'Pilar Peña Amaro

${ }^{2}$ Juan García López

${ }^{3}$ Yolanda Lupiáñez López

${ }^{3}$ Encarna Figueroa Magaña

${ }^{3}$ María Gámiz Magaña

${ }^{3}$ Ana Trujillo Abad

${ }^{1}$ Doctora. Licenciada en Enfermería y Licenciada en Antropología Social y Cultural. Profesora Titular Universidad de Jaén.

${ }^{2}$ Diplomado en Enfermería. Especialista en Enfermería Médico-

Quirúrgica. Especialista en Salud Mental. Unidad de Nefrología. Hospital Médico-Quirúrgico "Ciudad de Jaén".

${ }^{3}$ Diplomada en Enfermería. Centro de Salud "Oliveros”. Almería.

Correspondencia:

Pilar Peña Amaro

C/ San Francisco Javier, 2, escalera derecha - 2B.

23006 Jaén

Tfno.: 636133574

E-mail: ppena@ujaen.es

\section{Educación para la salud renal en personas mayores desde un centro de atención primaria}

\author{
Renal bealth education older people \\ from a center primary care
}

\section{RESUMEN}

Realizamos un programa de educación para la salud enfocado a la prevención primaria y secundaria de la enfermedad renal en personas mayores desde un centro de atención primaria.

Ponemos un póster en la sala de espera del centro con los contenidos sobre los que queremos incidir (hábitos de vida renosaludables). Cuando el paciente acude a consulta evaluamos los conocimientos que tenía y los que ha adquirido y los comentamos por si hay preguntas o dudas. Las personas mayores asocian la ingesta de sal y algunos alimentos (mariscos) con problemas renales, y como un factor de protección la ingesta de agua.

Creemos que en los/as ancianos y aún más en las poblaciones de riesgo (hipertensos y diabéticos) debería haber una mayor información sobre las conductas o hábitos saludables renoprotectores.

\section{PALABRAS CLAVE}

Educación para la salud, riñón, personas mayores, hábitos renosaludables.

\section{$S U M M A R Y$}

We make a program of education for the health focused on the primary and secondary prevention of the renal disease in major persons from a center of primary care.

We put a poster in the waiting room of the center with the contents we want to affect (habits of healthy life reindeer). When the patient enters to consultation we will evaluate the knowledge on that tape-worm and those he has acquired and we ask him/her if there are questions or doubts. The major persons associate the ingestion of salt and some food (seafood) with kidney problems and as a protection factor the water ingestion.

We believe that in the elders and furthermore in the populations of risk (hypertense and diabetic) there should be a major information about the conducts or healthy habits reindeer.

\section{KEY WORDS}

Education for the health, kidney, major persons, habits healthy reindeer.

\section{INTRODUCCIÓN}

La enfermedad renal (ER) se considera uno de los principales problemas de salud pública del siglo XXI. Más de 4 millones de personas (una de cada diez) se ven afectadas por ella, de hecho hay 40.000 personas que están en tratamiento sustitutivo (y se espera que esta cifra se duplique en los próximos diez años) ya que se están produciendo una entrada anual de 6.000 personas. Además, el aumento del envejecimiento progresivo de la población así lo están avalando (recordemos que el máximo de la función renal se alcanza a los 30 años y que a partir de ahí se inicia un deterioro orgánico de un $1 \%$ de la función renal).

Podemos afirmar que cada dos horas hay un nuevo paciente en diálisis. Esto nos hace valorar la importancia que está adquiriendo esta patología. 
110 Es necesario aumentar la conciencia que existe sobre la ER y sobre sus graves consecuencias (sobre todo a nivel cardiovascular) y llamar de esta manera la atención sobre la urgente necesidad global de realizar una detección temprana y procurar una prevención de esta enfermedad. Se ha comprobado que uno de cada nueve individuos aparentemente saludables, exhibe alguna forma de ER. Cuando esta se diagnostica, suele llevar ya un tiempo de evolución e incluso los tratamientos que se siguen pueden a veces no ser los más adecuados, por lo que la ER se va extendiendo y haciéndose más severa. Es necesaria la prevención y el freno a la progresión de la insuficiencia renal (IR).

Si bien se desconocen la mayoría de los factores que determinan que se produzca la destrucción del tejido renal, sí que se conocen algunas de las causas que la provocan (1), y entre ellas vamos a citar las siguientes:

1. Congénitas. (El 30\% de los familiares de pacientes con patología renal crónica presentan alteraciones que los hacen vulnerables para desarrollar algún grado de IR (la poliquistosis y el síndrome de Alport son los más corrientes).

2. Las glomerulonefritis.

3. Lesiones intersticiales. Infección urinaria. Reflujos vesico ureterales. Litiasis renal.

4. Vasculares (nefropatía aterosclerótica y enfermedad ateroembólica).

5. Sistémicas: diabetes, lupus Eritematoso, hipertensión arterial y amiloidosis.

6. Tóxicos (2): Aunque existen otros, nosotros por la población que estamos estudiando, a los que vamos a prestar especial atención es a los fármacos. Hay varios grupos: el paracetamol ( $\tan$ frecuente en su uso y que actúa por efecto acumulativo). Aspirina, iboprufeno. Los antibióticos (3) algunos de forma directa (aminoglucósidos, cefalosporínas, vancomicina, tetraciclina o anfotericina B) y otros por ser nefrotóxicos (sulfamidas, penicilinas, rifampicina...). Los AINE, antineoplásicos, contrastes, inmunosupresores, antirreumáticos, y en medicinas alternativas, el ácido aristolóquico ("raíces chinas"), Efedra, fruta de estrella, vitamina C, Uña de gato, etc.). en:

Los factores influyentes en la ER podríamos clasificarlos

1. Factores modificables, como son la hipertensión arterial, la diabetes mellitus, la hipercolesterolemia y la hipertrigliceridemia.

2. Factores no modificables, como pueden ser la edad o la raza.

En cuanto a la edad vamos a recordar los cambios que se producen en el sistema renal de las personas mayores por el propio proceso de envejecimiento y que son:

- La función de filtración renal (que desciende un $40 \%$ desde los 20 hasta los 90 años).
- El flujo de la sangre hacia el riñón disminuye en un 111 $53 \%$, variando de $600 \mathrm{~mL} / \mathrm{min}$ a $300 \mathrm{~mL} / \mathrm{min}$.

- La reabsorción de glucosa desciende en un 43\% (pudiendo aparecer glucosa en la orina).

- Hay una menor capacidad para reabsorber el sodio, y por consiguiente se pierde mayor cantidad de agua (lo que origina una predisposición a la deshidratación).

- Hay una menor capacidad de excreción de urea (con la consiguiente tendencia a acumular en sangre esta sustancia tóxica).

- Hay una disminución moderada en la capacidad de concentración de la orina.

- Y también existe una respuesta a las sobrecargas ácidas o básicas retrasadas y/o prolongadas.

\section{JUSTIFICACIÓN DE UN PROGRAMA DE PREVENCIÓN $(4,5)$ :}

En primer lugar comprobamos que la enfermedad renal cumple con las premisas (digamos necesarias) para realizar un programa de prevención. Así:

1. Es una enfermedad de alta prevalencia.

2. Los efectos de la enfermedad son importantes.

3. La enfermedad es fácilmente detectable y prevenible.

4. El coste del screnning y de la prevención es menor que el coste de la enfermedad (análisis de coste/beneficio).

Puesto que vemos la necesidad de realizarlo hay que plantearse cuáles son los objetivos de ese programa que va enfocado a mejorar la salud renal:

1. Promover la educación para la salud y los hábitos de vida saludables en la población en general.

2. Promover la integración de los cuidados de salud renal a los servicios de atención primaria en salud en todas las estructuras de atención medica.

3. Reconocer precozmente la enfermedad renal en las poblaciones de riesgo (hipertensos, diabéticos, polimedicados y personas mayores).

4. Optimizar el cuidado de los pacientes en todas las etapas de la enfermedad renal.

5. Difundir en los medios de comunicación y en los centros de salud los factores de riesgo de ERC y de cuáles son las conductas reno saludables.

6. Crear un sistema de identificación de pacientes.

Las medidas de prevención las estamos aplicando en los diferentes niveles:

1. Prevención primaria. Detectando las poblaciones de riesgo de presentar ERC (añosos, obesos, diabéticos, hipertensos, dislipémicos, fumadores). En estas poblaciones se debe realizar un tratamiento adecuado de los factores de riesgo y solicitar exámenes que permitan una detección temprana de la ER dada la concomitancia del riesgo de esta con la HTA (7) y la diabetes mellitas (DM). 
Peña Amaro, P.; García López, J.; Lupiáñez López, Y.; Figueroa Magaña, E.; Gámiz Magaña, M.a; Trujillo Abad, A.: EDUCACIÓN PARA LA SALUD RENAL EN PERSONAS MAYORES DESDE UN CENTRO DE ATENCIÓN PRIMARIA

2. Prevención secundaria. Más destinada a enlentecer la progresión de la enfermedad renal en quienes ya la presentan, evitando a su vez las complicaciones cardiovasculares.

3. Prevención terciaria. Que continúa con la anterior pero requiere ya de un equipo multidisciplinar de prediálisis que permite mejorar las condiciones de ingreso a tratamiento dialítico.

Como la ER (5) es frecuentemente asintomática por largos periodos de tiempo y sus únicas manifestaciones pueden ser alteraciones en el examen de orina o creatinina plasmática, tomaremos de entrada otras medidas también para detectarla como son, la medición de la tensión arterial, la de la glucemia en diabéticos, la toma de medicación hipolipemiante, o el uso de medicación renoprotectora que bloquean el eje renina angiotensina. Sabemos que la mayor parte de la población que se diagnostica de ERC es anciana. En un estudio realizado por Wetzels y cols. (6), en ancianos sanos, demuestran que en la mayoría de ellos, la única manifestación de ERC es el hallazgo de un filtrado glomerular disminuido que no se acompaña de otras alteraciones de daño renal salvo en una pequeña proporción de casos que presentan proteinuria (7). Por esto es tan importante un programa de prevención en mayores y en mayores de "riesgo".

Los instrumentos más importantes con los que contamos las enfermeras son la formación y la educación. Con nuestros programas de salud podemos actuar en la población que tenemos a nuestro cargo (8).

En cuanto a las ER producidas por lesiones intersticiales, haremos mas hincapié sobre todo en las infecciones urinarias recidivantes. Un tratamiento correcto y un diagnóstico precoz de las mismas, podrían evitar le lesión renal. Aunque son muy frecuentes, no se diagnostican siempre, debido a que sus síntomas son más de tipo general (o incluso no los presenta). Pero está claro que podremos prevenirla si controlamos los factores que las predisponen.

En caso de detección de la infección de vías urinarias (IVU) haremos un especial hincapié en el control y seguimiento correcto por parte del profesional sanitario cualificado (9).

En cuanto a la prevención de la ER en personas mayores que tienen o han tenido cálculos renales, la prevención pasaría en primer lugar por realizar las exploraciones correspondientes para conocer el tipo de cálculo que la ha producido y actuar, bien en la pauta dietética para corregirla, bien en la pauta medicamentosa. Por supuesto con un control posterior tanto de la dieta como del tratamiento.

La prevención en las enfermedades hereditarias habría que hacerla en primer lugar detectando los grupos de riesgo (los portadores, evitando tener descendencia con una buena planificación familiar) y en segundo lugar con un estudio familiar de todos los portadores.

En uno de los campos que más podemos actuar (y de hecho así es) es en el de la prevención de ER por causas vasculares, y aquí la hipertensión arterial (10) y la diabetes 111 son las principales protagonistas.

Aunque ambos tienes sus programas específicos en nuestras consultas de enfermería para su control, recordemos que la prevención que podríamos hacer sería actuar en sus diversos componentes modificables:

1. Sobrepeso u obesidad.

2. Ingesta de sal.

3. Ingesta de alcohol.

4. Tabaco.

5. Sedentarismo.

Eso, y detectarla precozmente, haciendo especial hincapié en la utilización de medidas higiénico dietéticas adecuadas, seguimiento y control de la TA de forma periódica, adhesión al tratamiento del paciente y conocimiento del proceso, forma de acción de la medicación, efectos secundarios y precauciones y no alterar la prescripción.

Los diabéticos merecerían un capitulo solo para ellos ya que es el factor que más influye (1/3 de los pacientes). Igualmente, nuestras actividades van enfocadas a los controles periódicos en los diabéticos no solo de la glucosa (importantísimo), sino también de la proteinuria y de la dieta (control de peso y dieta específica) así como en los hábitos de vida saludables incluyendo el ejercicio físico y la forma de realizarlo.

Las ER producidas por patologías multisistémicas no tienen más prevención que un correcto tratamiento de la enfermedad de base para evitar o retrasar la nefropatía. Después, con una educación sanitaria adecuada, se concienciará a la persona sobre el proceso, la medicación, los riesgos, etc.

Aunque no hay una sola prevención "controlada" de las alteraciones renales sí que podemos hacer un plan de prevención:

1. Controlando la TA de la población (1-2 veces al año o aún más veces si es población de riesgo, hipertensos, diabéticos o embarazadas) (10). El control de la tensión arterial debe ser estricto con valores menores a 130/80 mm, y si existe proteinuria los valores deben ser menores a $125 / 75 \mathrm{mmHg}$.

2. En caso de diabetes, el objetivo es la euglicemia, recomendándose valores de hemoglobina glicosilada normales (menores de 7\%).

3. El ejercicio físico moderado, y supresión total de alcohol y tabaco.

4. Control adecuado de peso la dieta hipocalórica Una dieta con cierta limitación de proteínas es beneficiosa: limitar carnes rojas, cerdo, algunos pescados o fiambres. Reducción de la sal.

5. Los hábitos alimenticios saludables previenen no solo las patologías renales sino tambien algunas causantes de ellas como la HTA, diabetes mellitus, cardiovasculares o la obesidad. Normalización de los niveles séricos de colesterol y triglicéridos no solo por productores de patologías ateroembólicas sino tambien como elementos favorecedores de la progresión renal. 


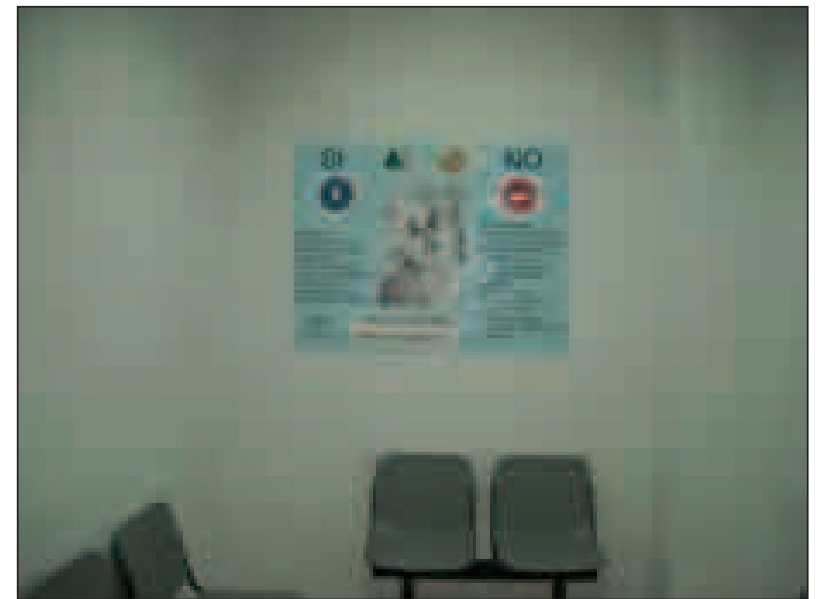

Fig. 1. Póster en sala de espera del centro.

6. Realizando una analítica sencilla de orina al año (anormales, sedimento y vigilando principalmente las proteinuria) a personas mayores, y más frecuente si se observa algún signo o molestia urinaria. Tambien determinaciones de creatinina y lípidos en sangre.

7. Insistencia en la no automedicación (ni analgésicos, antiinflamatorios, diuréticos ni laxantes...).

La prevención no puede evitar la enfermedad renal, pero puede retrasar el curso de la misma y mejorar la morbi-mortalidad (principalmente por la causa cardiovascular). Por eso es tan importante.

\section{MATERIAL Y MÉTODO}

Hemos colocado un póster (Fig. 1) en la sala de espera de nuestro centro de salud (en las consultas de enfermería). Contiene una imagen de una mujer mayor (llamada "Renata" y cuya característica principal es que los ojos tienen la forma de riñones y la nariz son las vías urinarias ) y un lema que dice: "Renata te dice cómo mirar por tus riñones". A la izquierda de la imagen hay una señal de dirección obligatoria y un círculo que dice "Sî" y a la derecha una señal de dirección prohibida y otro círculo que contiene un "No". Por debajo del "sí" hay conductas sanas y recomendables y por debajo del "no" las que no lo son.

Cuando los pacientes entran a consulta les preguntamos sobre el póster (pasándoles un cuestionario de evaluación) estableciendo así una comunicación y una vía para evaluar y comentar conocimientos y las acciones apropiadas para evitar o minimizar daños renales.

Cuando terminamos nuestra charla les damos (si lo desean) las recomendaciones por escrito.

\section{RESULTADOS OBTENIDOS}

El $6 \%$ no se han fijado en el cartel ni lo han leído.

$\mathrm{El}$ perfil de los pacientes es el siguiente:

Con unos niveles de estudios básicos el 56,2\%, hombres el $43,7 \%$ y fumadores/as el $6 \%$.

La edad media de los encuestados es de 61,2 años, siendo su distribución la siguiente:

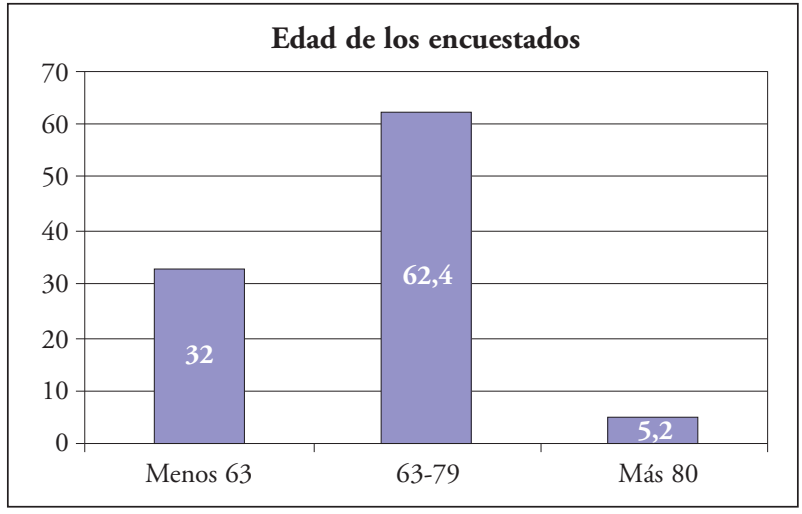

En cuanto a sus hábitos en la ingesta de líquidos, toman:

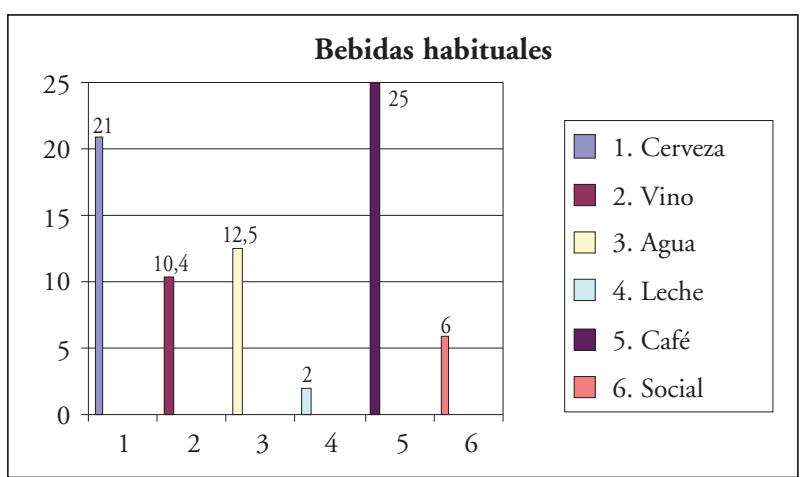

En la pregunta acerca de sus hábitos relacionados con la ingesta de sal, vemos:

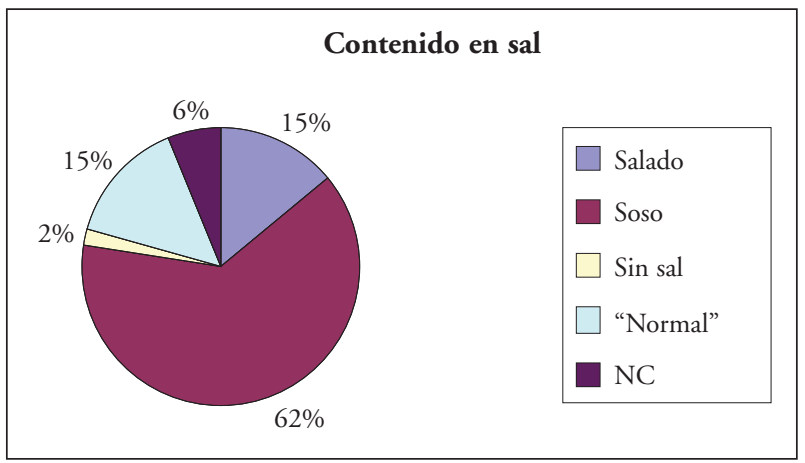

Sus hábitos higiénicos son en un $83,3 \%$ de ducha y un $14,5 \%$ se lavan "por partes" (la frecuencia es un dato que eluden).

Las patologías que tienen son:

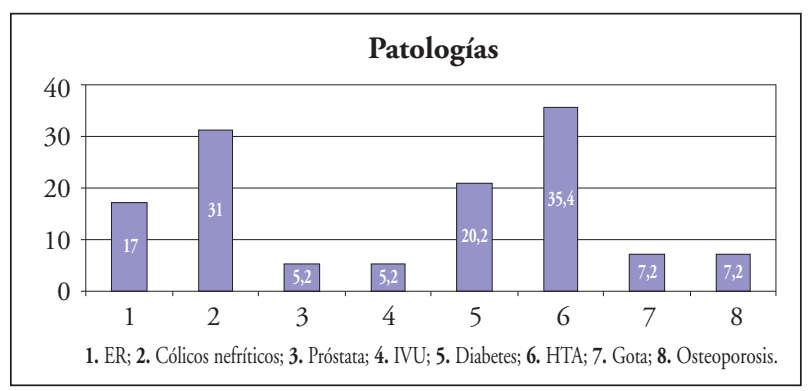


Peña Amaro, P.; García López, J.; Lupiáñez López, Y.; Figueroa Magaña, E.; Gámiz Magaña, M.a; Trujillo Abad, A.: EDUCACIÓN PARA LA SALUD RENAL EN PERSONAS MAYORES DESDE UN CENTRO DE ATENCIÓN PRIMARIA

El peso medio es de 78,5 kilos y su distribución es la siguiente:

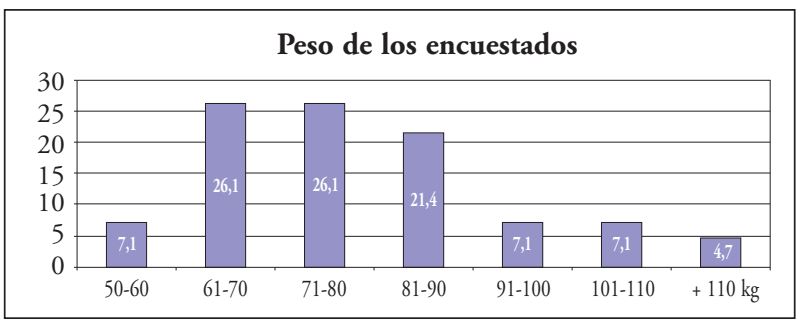

Controlan su peso el 85,4\% y índice de masa corporal (IMC) siguiendo los criterios de la SEEDO son:

\begin{tabular}{|l|c|}
\hline Normopesos & $18,5 \%$ \\
\hline $\begin{array}{l}\text { Sobrepeso (grado I) } \\
\text { (IMC entre 25-26,9) }\end{array}$ & $14,8 \%$ \\
\hline $\begin{array}{l}\text { Sobrepeso (grado II) } \\
\text { (IMC entre 27-29,9) }\end{array}$ & $22,2 \%$ \\
\hline $\begin{array}{l}\text { Obesidad (tipo I) } \\
\text { (IMC entre 30-34,9) }\end{array}$ & $33,3 \%$ \\
\hline $\begin{array}{l}\text { Obesidad (tipo II) } \\
\text { (IMC entre 35-39,9) }\end{array}$ & $7,4 \%$ \\
\hline $\begin{array}{l}\text { Obesidad mórbida (tipo IIII) } \\
\text { (IMC entre 40-49,9) }\end{array}$ & $3,7 \%$ \\
\hline
\end{tabular}

En relación a la glucosa la miden o la controlan el $47,9 \%$ y la TA un $85,4 \%$ de los casos.

En cuanto a la actividad física caminan un $62,5 \%$ y no hacen nada un 20,8\%; gimnasio en un $6,2 \%$ y el resto fútbol, bicicleta o nadar de forma poco significativa. El tiempo que invierten en esta actividad oscila entre media hora y una hora diaria en un $14,5 \%$.

En cuanto a la medicación que toman son polimedicados (con más de cinco fármacos) en un 58,3\%, con un medicamento solo el 10,4\%, con dos medicamentos el 16,6\%. Solo toman analgésicos si tienen dolor en un 2,08\%.

Cuando les pasamos el cuestionario para ver qué conceptos recuerdan de los que se exponían en el póster de la sala de espera y que eran beneficiosos para el riñón, vemos que contestan:

\begin{tabular}{|l|r|l|r|}
\hline Beneficioso & $\%$ & Dañino & $\%$ \\
\hline No beber es malo & 4,1 & Alcohol & 54,1 \\
Mucho peso & 2 & Café & 37,5 \\
Hay que tomarse la TA & 2,08 & Proteínas & 2 \\
Hay que medir la glucosa & 2 & Lácteos & 29,1 \\
Hay que beber agua & 93,7 & El sedentarismo & 64,5 \\
\hline
\end{tabular}

\begin{tabular}{|l|r|l|r|}
\hline Beneficioso & \multicolumn{1}{|c|}{$\%$} & Dañino & $\%$ \\
\hline Hacer ejercicio & 77 & Dulces/chocolate & 6,2 \\
No tomar medicinas sin receta & 2 & Grasas & 45,8 \\
Tomar frutas/verduras & 25 & No beber agua & 27 \\
Buena higiene & 8,3 & La sal & 37,4 \\
Caminar & 2 & Comer mucho & 2 \\
Comer carne & 2 & Te & 6,2 \\
No tomar café & 16,6 & Beber poca agua & 20,8 \\
No tomar sal & 25 & Azúcares/harinas & 2 \\
Comer pescado & 6,2 & Pescado & 8,3 \\
No comer espinacas & 2,08 & Medicinas & 0,2 \\
No comer grasas & 35,4 & No contestan & 2 \\
No beber alcohol & 12,5 & & \\
No fumar & 16,5 & & \\
Seguir dieta sana & 20,8 & & \\
No tomar mucho azúcar & 4,1 & & \\
No contestan & 4 & & \\
\hline
\end{tabular}

\section{DISCUSIÓN}

Hay varias cosas que nos han llamado la atención en la realización de nuestro trabajo. Las personas mayores mayoritariamente tienen el concepto de que la sal es mala para el riñón, pero no que el sobrepeso, la falta de ejercicio y la polimedicación lo sean. No asocian diabetes o hipertensión con potencial daño renal, aunque sí lo hacen con los cólicos nefríticos y "algunos alimentos" como ciertos "pescados" (mariscos), pero no con las carnes o los huevos.

A la hora de evaluar sus conocimientos nos ha llamado mucho la atención la "forma" de contestar. Aunque nosotros les pedíamos que nos dijeran cosas beneficiosas para el riñón, nos han contestado en positivo (es decir, citando cosas que son beneficiosas como por ejemplo beber agua, hacer ejercicio o llevar una dieta sana) una minoría. Sus respuestas estaban en relación con lo que no es bueno... (nos decían: no es bueno tomar café, no es bueno comer sal, no es bueno comer grasas, no es bueno beber alcohol o no es bueno fumar).

Cuando les preguntábamos por cosas nocivas contestaban en positivo de forma más mayoritaria y centrando su interés en el sedentarismo, la ingesta de grasas y el alcohol, y solo contestaban en negativo que es malo no beber agua.

Nuestra población recordaba más lo que no es bueno que lo que sí lo es.

\section{CONCLUSIÓN}

Una educación sanitaria adecuada puede retrasar o minimizar los problemas renales que se pueden presentar en la tercera edad, tanto si existen otras patologías (como la diabetes o la hipertensión) como si la disminución de la función renal es la fisiológica propia del envejecimiento. 
Peña Amaro, P.; García López, J.; Lupiáñez López, Y.; Figueroa Magaña, E.; Gámiz Magaña, M.a; Trujillo Abad, A.: EDUCACIÓN PARA LA SALUD RENAL EN PERSONAS MAYORES DESDE UN CENTRO DE ATENCIÓN PRIMARIA

\section{BIBLIOGRAFÍA}

1. Palma Alfonso. Causas de la IRCT. En: LLack y Valderrabano. IRCT (diálisis y trasplante renal. Vol. 1). Madrid: Editorial Norma, 1997, pp. 23-46.

2. Rodríguez Jornet A, Towar JL, Ferrea Piedra L. Complicaciones nefrológicas de la intoxicación por plomo. Med Clin (Barcelona) 1984; 83: $164-6$.

3. Revert Torrellas L. Antibióticos e insuficiencia renal. Forum 164. Marzo 89.26.

4. Ronda MJ. Prevención de la IR. Atención primaria de enfermería. En: Cuidados de enfermería en la IR. (Joan Andrés y Carme Fortuny). Madrid: Editorial Gallery/healthCom S.A., 1993, pp. 43-51.

5. Martínez Maldonado M. Prevención de la IRC. En: Llack y Valderrabano. IRCT (diálisis y trasplante renal .Vol. 1). Madrid: Editorial Norma, 1997, pp. 742-56.
6. Wetzels JF, Willems HL, den Heijer M. Age and gender-specific reference values of estimated glomerular filtration rate in a Caucasian population: Results of the Nijmegen Biomedical Study. Kidney Int 2008; 73 (5): 657-8.

7. Hernando Avendaño L, López Novoa J. Glomerular filtration and renal blood flow in the aged. En: Macías Núñez JF, Cameron S (Eds). Renal function and disease in the elderly. London: Butterworth, 1987, pp. 27-48.

8. Campaña de prevención de la IR. SEDEN 1985.

9. Guía clínica de las infecciones urinarias. Hospital Marques de Valdecilla. Año 2006.

10. Molina Díaz y Martí Canales. Traducción del séptimo informe del Joint Nacional Committee sobre prevención, detección, evaluación y tratamiento de la hipertensión arterial. Arch Interm Med 1997; 157: 2413-66.

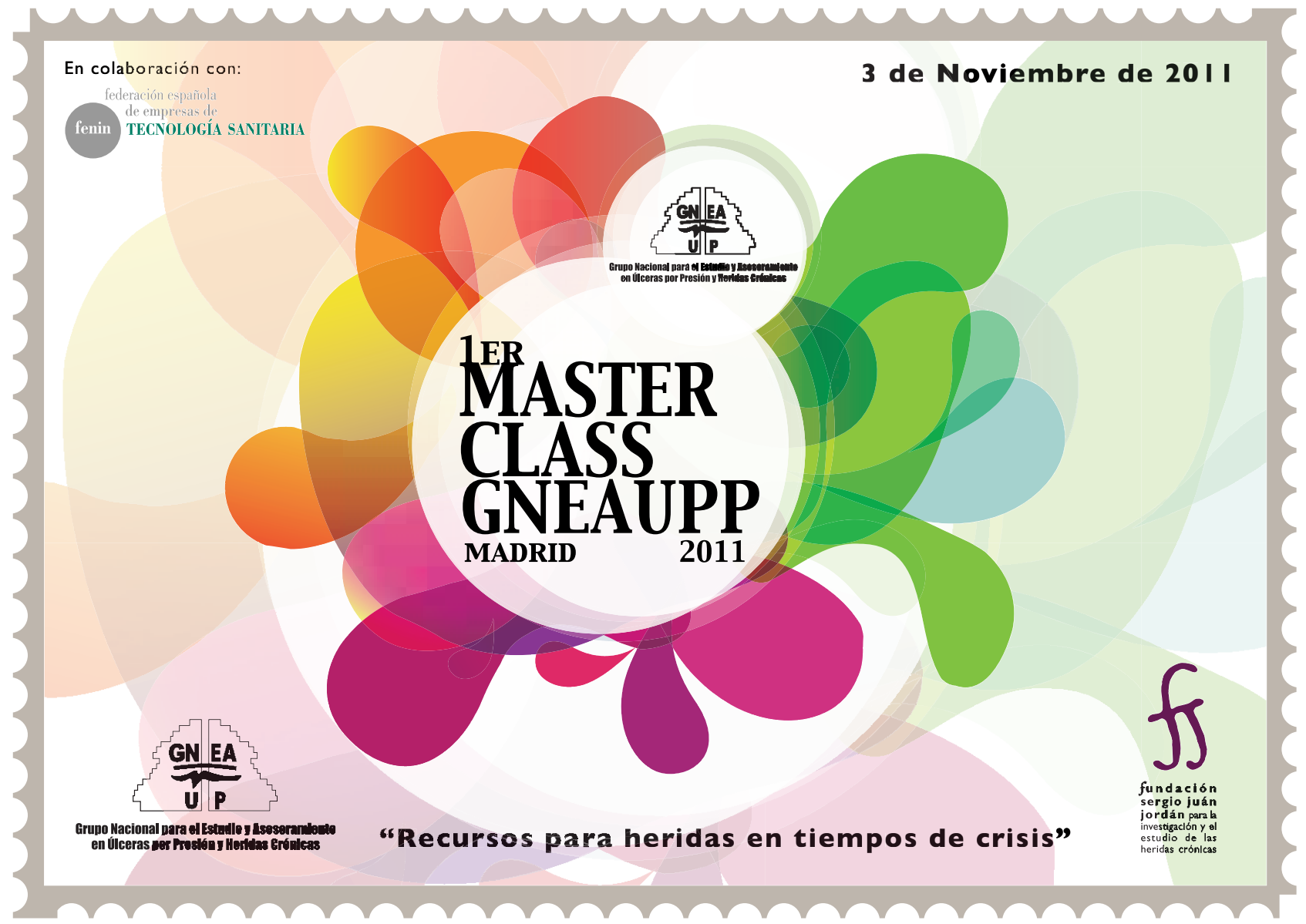

